\title{
Coparenting Intervention for Expectant Parents Affects Relationship Quality: A Pilot Study
}

\author{
Anna C. Philipp, ${ }^{1}$ Jin-kyung Lee, ${ }^{2}$ Tanja A. Stamm, ${ }^{3}$ Michael Wininger, ${ }^{4}$ \\ Wilfried Datler ${ }^{4}$ and Nestor D. Kapusta ${ }^{1}$
}

\begin{abstract}
${ }^{1}$ Department for Psychoanalysis and Psychotherapy, Medical University of Vienna, Vienna, Austria
${ }^{2}$ Edna Bennett Pierce Prevention Research Center, Pennsylvania State University, University Park, PA, USA

${ }^{3}$ Section for Outcomes Research, Center for Medical Statistics, Informatics, and Intelligent Systems, Medical University of Vienna, Vienna, Austria

${ }^{4}$ Research Unit Psychoanalysis and Education of the Department of Education, University of Vienna, Vienna, Austria
\end{abstract}

Research has shown that the transition to parenthood is a particularly challenging period of life which is often associated with a decline in relationship quality and an increase in mental health problems. Emerging parents often experience difficulties in coping with new tasks and challenges in the relationship, resulting in inadequate mutual support, stress, conflicts and even depressive symptoms. To support expectant parents in establishing an effective and strong coparenting alliance, we have employed an educational coparenting intervention to teach important coparenting skills. The intervention was a non-randomized case-control study with 126 expectant parents. The intervention group participated in a five-session intervention, whereas the control group received an information booklet and had an optional meeting postpartum. The purpose of this study was to ease the transition to parenthood in order to prevent postpartum conflict and depression. Parents in the intervention group ( $n=34$ couples) showed significantly fewer conflicts postpartum than before $(Z=-3.28, p=0.00)$, and scored better in postnatal delegated dyadic coping $(\beta=$ $\left.0.25, p=0.00, R^{2}=0.32\right)$, a form of mutual support. Neither the intervention group $(Z=-0.83, p=$ $0.40)$ nor the control group $(Z=-0.86, p=0.38)$ showed a significant increase in depression scores after childbirth. Although conflicts during the transition to parenthood declined and postnatal delegated dyadic coping strengthened, the study design does not allow to draw conclusion on group effects. Nevertheless, the promising results of this pilot intervention are a base for future studies.

Keywords: conflict; coparenting; intervention; relationship quality; transition to parenthood Tohoku J. Exp. Med., 2020 September, 252 (1), 33-43.

\section{Introduction}

The transition to parenthood is an emerging field of interest in scientific research. The birth of one's first infant leads to a fundamental change in an individual's life. On the one hand, it is a joyful major life event; on the other hand, it is associated with stress and strain (Brotherson 2007; Deave et al. 2008; Diem-Wille 2018). The first months after birth are perceived as particularly stressful because a baby requires almost constant attention. New parents have to cope with a number of stressors, such as tiredness; lack of time together as a couple; and increased conflicts, chores, housework, and individual stress, includ- ing postpartum depression, just to name a few (Tomlinson 1996; Condon et al. 2004; Brotherson 2007). Some couples may grow through such a challenging experience and strengthen their relationship. For a significant proportion of new parents, however, particularly for those with prebirth relationship or individual problems, the transition to parenthood often triggers a shift in relationship quality that leads to a decline in relationship satisfaction, an increase in conflicts, and the possible development of psychopathological symptoms (Cowan et al. 1985; Moss et al. 1986; Condon et al. 2004).

Postpartum depression is a major mental health problem that affects women worldwide. About $10-30 \%$ of new

Received January 6, 2020; revised and accepted August 3, 2020. Published online August 28, 2020; doi: 10.1620/tjem.252.33.

Correspondence: Anna C. Philipp, Department of Psychoanalysis and Psychotherapy, Medical University of Vienna, 18-20 Währinger

Gürtel, Vienna 1090, Austria.

e-mail: anna.christina.phillip@gmail.com

(C)2020 Tohoku University Medical Press. This is an open-access article distributed under the terms of the Creative Commons Attribution-NonCommercial-NoDerivatives 4.0 International License (CC-BY-NC-ND 4.0). Anyone may download, reuse, copy, reprint, or distribute the article without modifications or adaptations for non-profit purposes if they cite the original authors and source properly.

https://creativecommons.org/licenses/by-nc-nd/4.0/ 
mothers suffer from this mood disorder, and many women show postnatal symptoms of anxiety and stress (Miller et al. 2006; Paulson and Bazemore 2010; O'Hara and McCabe 2013). Perinatal depression begins during pregnancy and/ or within four weeks after the child's birth (American Psychiatric Association 2013). The World Health Organization (2010) considers the time of onset of this mood disorder to be as long as one year postnatal. Postpartum depression often emerges when a mother feels overwhelmed or lacks social support (O'Hara and McCabe 2013). Poor marital quality and low social support are strong forecasters of postpartum depression in women (O’Hara and Swain 1996). Similarly, marital conflicts and poor communication are more often observed in depressed women than in nondepressed women (Johnson and Jacob 1997; Parfitt and Ayers 2009). Existing literature emphasises the presence of paternal depression and its link to negative child outcomes (Condon et al. 2004; Ramchandani et al. 2005; Kapusta et al. 2017). According to Cowan and Cowan (1995), parents' well-being or distress during the family-making process influences children's cognitive development, success with peers, and behaviour problems in early elementary school. Family communication and family time, on the other hand, as well as support networks, are known to be effective buffers against stressful life events (Black and Lobo 2008). If parents were able to support each other as well as to overcome difficulties during the transition to pranethood, they could create a positive and harmonious atmosphere, which is the basis for healthy infantile development (Brotherson 2007).

Dyadic coping, a form of mutual support, may be understood as such a buffer that needs to be developed between partners. Delegated dyadic coping is a positive way to help a partner cope with stress by taking over some of the partner's tasks (Falconier and Kuhn 2019). Studies have shown positive associations between this kind of support and constructive conflict resolution as well as relationship satisfaction in couples (Falconier et al. 2013; Randall et al. 2015). More recently, Molgora et al. (2019) highlighted that couples with high levels of delegated dyadic coping report high levels of couple adjustment.

Coparenting is another important concept related to parenthood. Parenting behaviour focuses on infant care, parental responsiveness, sensitivity, and the promotion of children's cognitive stimulation (Doss et al. 2014). In contrast, coparenting is defined as the "extent to which spouses function as parents or adversaries in their parenting roles" (Gable et al. 1994), or as the "way that parents work together in their roles as parents" (Feinberg 2002). Research has highlighted that coparenting support has a more powerful impact on parental adjustment and postnatal depression than other couple support approaches. Furthermore, the improved quality of coparenting has a positive effect on child outcomes (Feinberg 2002). Recognizing that coparenting alliances are important to the well-being of both couples and their children, couple- focused interventions to ease the transition to parenthood are warranted and needed. Such interventions should take place as early as possible, even during the early stages of pregnancy, because the early years of a child's life are crucial to their later outcomes and because parents are quite open to changes in the time around the birth of their child (Elliot et al. 2000; Feinberg 2002).

A few universal preventive intervention programmes with evaluation components that ease the transition to parenthood and improve coparenting have already been implemented in Anglo-American and Asian regions (Shapiro and Gottman 2005; Fagan 2008; Feinberg and Kan 2008; Doss et al. 2014; Jones et al. 2018; Takeishi et al. 2019). Such intervention programmes enhance couples' coparenting skills, promote harmonious relationships, and reduce the overall level of stress and conflict during the family formation period. They also aim at preventing the development of postpartum depression. In other words, providing help for expectant mothers and fathers can lead to important mental health and developmental benefits for them and, in the long run, for their children (Cowan and Cowan 1995; Feinberg et al. 2009; Jones et al. 2018). Therefore, it is important to work with both mothers and fathers in the antenatal and postnatal periods (Hanington et al. 2012; Lee et al. 2018).

Such an intervention in the German-speaking world would enhance existing prenatal education. Pregnant women in Austria currently receive care from health professionals including gynecologists and midwives (KöenigBachmann et al. 2019). In addition to supporting obstetrics, midwives offer pregnant women preventive medical examinations, birth preparation, gymnastics exercises, and extensive breastfeeding support. However, men are typically excluded from these forms of support. In this study, we present a form of support for expectant parents in the German-speaking world that actively includes both partners within a couple. This support takes the form of a psychoeducational coparenting intervention that helps expectant parents to establish an effective and strong coparenting alliance. Through this intervention, parents-to-be learn how to work together effectively to make the challenging transition to parenthood without experiencing major side effects. This intervention was designed as a pilot of an abbreviated version of the Family Foundations programme (Feinberg 2002), adapted to meet the needs of the European, especially Austrian culture (Belsky 1984; Cowan et al. 1985; Cowan and Cowan 1988, 1995; Harms 2008). We expected that the coparenting intervention would (1) strengthen a couple's relationship quality, (2) facilitate positive coparenting functioning as primary outcomes, (3) serve as a buffer against postpartum depression, and (4) facilitate mutual support, representing secondary outcomes. The goal of this pilot study was to see if an pre- and post-partum intervention shows significant results to prevent postpartum depression and conflict as well as to deliver scientific insights which can serve as starting points for future studies. 
As part of our analysis, we also considered genderspecific differences in coparenting functioning in anticipation of gender-specific adaptions in future studies.

\section{Material and Methods}

\section{Design and Participants}

This pilot study used a non-randomized, case-controlled design. The target group was comprised of pregnant women and their partners. To be eligible for participation in the study, an individual was required to be at least 18 years old, heterosexual, expecting its first child with the current partner, and to be sufficiently proficient in the German language in order to ensure homogenous cases (Song and Chung 2010). The study only included parents who were expecting their first child as a couple in order to reduce the impact of complexity in family processes. Couples expecting twins were excluded from all analyses to avoid familial clustering effects (Hanington et al. 2012).

\section{Procedures}

Participants were recruited via Viennese obstetricians, delivery clinics, pamphlets, social media advertisements, websites of birth-related organizations, word of mouth, and midwives. The option of participating in an informational evening was offered. Those who did not participate in the informational evening received information about the intervention via email. Couples were also informed that participation was entirely voluntary and that they could withdraw from participating at any time without reprisal. All participants provided written informed consent. Couples selfselected into the intervention or control group according to their available time and personal preference. Data were collected at two timepoints: before the start of the intervention during pregnancy and after the completion of the postnatal intervention. The study began in spring 2017 and ended in summer 2018. Fig. 1 illustrates the procedure for the study. One hundred and fifty-six expectant parents were recruited and assessed for eligibility. A total of 80 expectant parents chose to be in the intervention group, and 76 expectant parents opted for the control group. Twelve participants in the intervention group and 18 participants in the control group dropped out during the course of the study, leaving us with a total sample of 126 participants (see Fig. $1)$.

All couples who consented to participate in the experimental group received the five-session intervention, whereas the couples in the control group received a 40-page booklet and the option of participating in one meeting with the other parents in the control group postpartum. The postpartum meeting for parents of the control group served to exchange new experiences with each other. This meeting also constituted the second timepoint in the data collection process.

The intervention sessions were led by a psychologist, a psychiatrist, as well as by a midwife. The five sessions consisted of monthly lectures in the evening on the premises of the Medical University of Vienna and the University of Vienna. The whole intervention consisted of three prenatal and two postnatal sessions. Each session took about two hours. The content was conveyed via diverse approaches, including PowerPoint presentations, videos, handouts, discussions, worksheets, and homework tasks. The main contents of our coparenting intervention involved psychoeducation about baby blues and postpartum depression in mothers and fathers; dealing with conflicts and feelings of guilt, anxiety and worry; mutual support; coparenting functioning; expectations, wishes and hopes about parental roles and childrearing; communication patterns between partners; conflict and stress management; development of a secure attachment to the baby; child tempera-

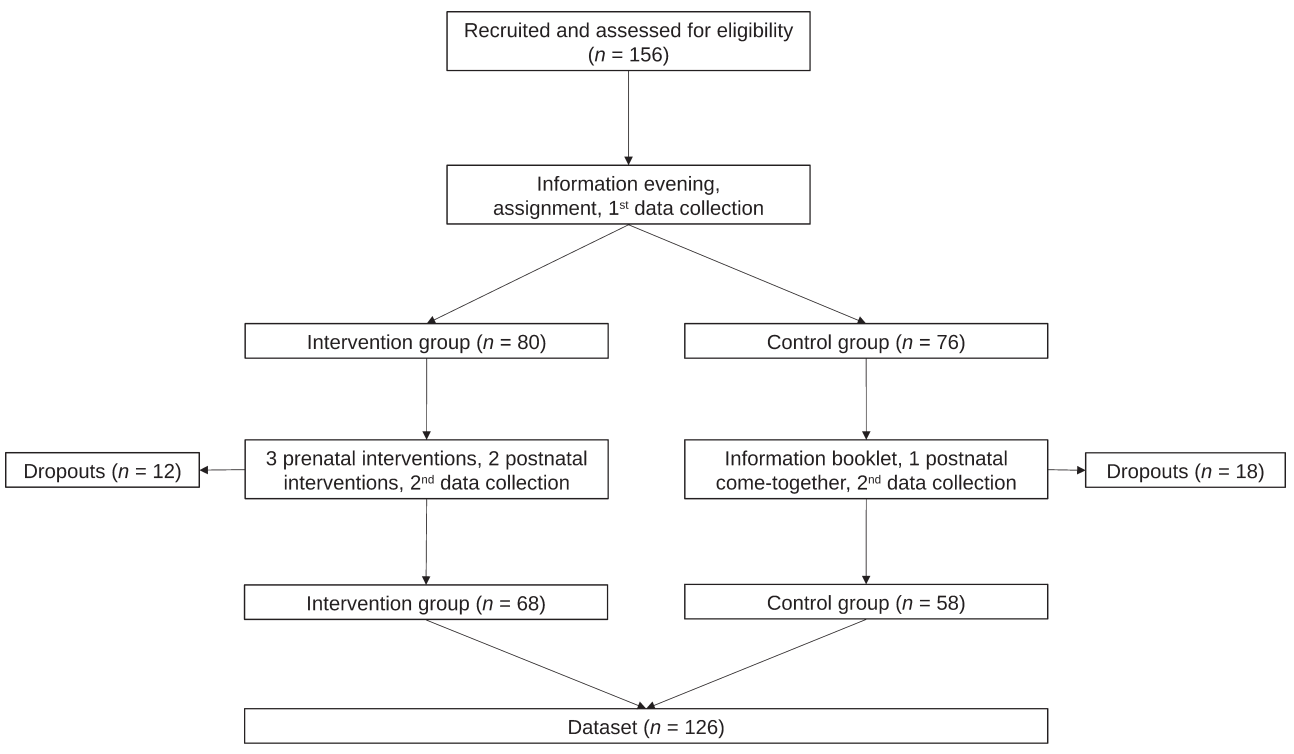

Fig. 1. Flow of participants through each stage of the pilot study. 
ment; the influence of one's own childhood experiences on the upbringing of one's child and the couple's sexuality and postpartum intimacy.

The booklet for the control group was specially developed for this study. It covers the same topics as the full intervention, but in a much reduced form. In particular, it addresses the meaning of coparenting, its components, and strategies for developing a strong coparenting relationship. The booklet also discusses mutual support, conflict and stress management, baby blues and postpartum depression, intimacy after childbirth, and attachment patterns of newborns. Furthermore, the control group received fewer worksheets than the intervention group.

\section{Measurements}

Questionnaires were administered at baseline (at the time of enrolment; women were in the second or third trimester of gestation on average) and at a mean of 13.34 weeks postpartum $(\mathrm{SD}=5.35)$. Self-report questionnaires with proven good psychometric properties as well as questionnaires assessing sociodemographic information were administered. We collected data about coparenting behaviour, the quality of a couple's relationship, the availability of mutual support, and postnatal depression. Sociodemographic information such as relationship status; highest level of education; type of employment and annual gross income as well as whether they sought help from a midwife or psychotherapist and whether women had complications during pregnancy or childbirth were also administered. Attachment patterns were also administered. The Relationship Questionnaire (RQ) (Bartholomew and Horowitz 1991) is a short and valid questionnaire designed to measure adult attachment. It consists of four vignettes, each describing a prototypical attachment pattern (secure, fearful-avoidant, preoccupied, and dismissing). Each participant was asked rate their degree of correspondence to each prototype on a 7-point Likert scale, resulting in a profile of the individual's attachment style. The RQ was administered at baseline. Education level was included as control variable because it is related to relationship quality (Tynes 1990; Wiik et al. 2009) and thereby affects a primary outcome.

To control participants' response behaviour for social desirability, the Social Desirability Scale was administered at baseline. It consists of six items measuring deception of others and self-deception and shows good internal and external validity as well as good reliability (Winkler et al. 2006). The analysis showed that neither the intervention group nor the control group gave socially accepted answers. On the scale, "deceiving others," the intervention group ( $n$ $=68 ; 34$ couples) showed a mean of $14.02(\mathrm{SD}=3.13)$ and the control group $(n=58 ; 29$ couples) showed a mean of $14.68(\mathrm{SD}=3.24)$. Similarly, on the scale of "self-deception," the intervention group had a mean of 15.20 (SD = 2.14 ) and the control group had a mean of 14.87 (SD = 2.44) with no significant intergroup differences (deceiving others, $\mathrm{p}=0.34$; self-deception, $\mathrm{p}=0.20$ ).

\section{Quality of relationship}

The Quality of Relationship Inventory (QRI) (Reiner et al. 2012) is a self-assessment questionnaire used to determine the quality of a couple's relationship and their satisfaction with the relationship. This questionnaire yields three scores: social support, depth, and conflict. It shows high criterion validity and its internal consistency ranges from $\alpha=0.82$ to 0.89 . It was administered at baseline and postpartum.

\section{Coparenting functioning}

The Coparenting Relationship Scale (CPRS) (Feinberg et al. 2012) consists of 35 items and measures important aspects of coparenting: coparenting agreement, coparenting closeness, exposure to conflict, coparenting support, coparenting undermining, endorsing partner parenting and division of labor. It demonstrates excellent internal consistency (Cronbach's $\alpha$ ranging from 0.81 to 0.89 ), good reliability, strong stability, good construct validity, and good inter-rater agreement. All seven subscales show good reliability as well. The original CPRS was translated into German, then back-translated into English, controlled in both cases by a native speaker. The resulting internal consistency of the German-language version had a Cronbach's $\alpha$ of 0.81 . This questionnaire was only administered postpartum.

\section{Depression}

The Edinburgh Postnatal Depression Scale (EPDS) (Cox et al. 1987) is a widely used self-assessment questionnaire based on 10 items. It is a validated screening tool that measures prenatal and postnatal depression and anxiety disorders in women and men. The EPDS has satisfactory reliability and good internal consistency (Cronbach's $\alpha$ of 0.87 ) (Robakis et al. 2016). It was administered at baseline and postpartum.

\section{Mutual support}

The Dyadic Coping Inventory (DCI) (Bodenmann 2008) is a 37-item questionnaire that measures whether a couple handles stress by supportive dyadic coping, joint dyadic coping, or delegated dyadic coping. While positive supportive dyadic coping occurs when one partner supports the other in coping with stress, delegated dyadic coping is when the supporting partner completely takes over the burdensome situation. Joint dyadic coping is characterized by shared problem-solving. Dyadic coping is known to be a very important predictor of the quality of a relationship. The psychometrics of the DCI are good. The internal consistencies of the subscales range from $\alpha=0.72$ to 0.92 (women) and 0.71 to 0.89 (men). This questionnaire was adminstered at baseline and postpartum.

\section{Statistical analysis}

The statistical analysis was carried out per protocol by 
excluding dropouts and considering only cases with completed data. The procedure was necessary because the CPRS, which measured a primary outcome, was only filled out at the postnatal time point. The final analysis was carried out with data from 126 participants.

Normality assumptions were checked using the Kolmogorov-Smirnov test. Intergroup differences were tested by a t-test or a nonparametric Mann-Whitney U-test. Outliers and the Levene test were checked before using t-tests. A Wilcoxon signed rank test was used to test prenatal and postnatal intragroup differences. A t-test and Fisher's exact test were used to analyse baseline group differences and dropout rates. An ANOVA was performed in order to explore the impact of education level on quality of relationship. Correlations were calculated using Spearman correlations. Ordinary least-squares regressions were used to test if intervention status was able to predict post-test scores of outcome variables, controlling for pre-test scores of the same outcome variables and other family or couple relationship characteristics at baseline. All tests were twotailed, and the significance level was set at $p<0.05$. Data were analysed using IBM $^{\circledR}$ SPSS $^{\circledR}$ Statistics.

\section{Ethical standards}

All procedures were performed in accordance with the guidelines for good clinical practice (GCP). According to GCP guidelines, an application for the local ethics committee approval was submitted and accepted. The ethics committee issued ID 2113/2016.

\section{Results}

\section{Sample Characteristics}

The pilot study consisted of 126 expectant Austrian parents who had been in their partnerships for an average of 7.29 years $(\mathrm{SD}=4.7)$, with a minimum of 1.16 years and a maximum of 22.00 years. At the time of recruitment, mothers-to-be were on average 32.2 years old $(\mathrm{SD}=3.9)$ and fathers-to-be were on average 34.74 years old $(\mathrm{SD}=5.1)$. Each participant was expecting its first child with the current partner and had sufficient German-language skills to fill out the questionnaires. Following sample descriptive statistics are shown in Table 1. Of the participants, 118 (93.7\%) were employed and 7 (5.6\%) were unemployed. The majority had an annual gross income of $€ 30.000$ $60.000(47.9 \%)$, as well as a university degree $(61.9 \%)$. Fifty percent were in partnerships where the partners had a joint place of residence (Table 1). Moreover, most of the expectant parents sought additional help parallel to the intervention, including so-called antenatal classes (83.3\%); support from a midwife, who offers preventive medical examinations, birth preparation, gymnastics exercises, and extensive breastfeeding support (84.1\%); and psychotherapeutic help (12.7\%). Some of the women had reported experiencing complications during pregnancy $(14.3 \%)$ or complications during delivery (30.2\%).

During initial testing, which took place at the time of recruitment, most pregnant women were in the second trimester (49.2\%). The subquent testing took place when the newborns were an average of 13.46 weeks old $(\mathrm{SD}=5.35)$, with a range of 6.00 weeks to 28.00 weeks. In the postnatal period of testing, 11 couples $(37.9 \%)$ out of 29 couples in the control group participated in the postpartum meeting, while the other 18 couples $(62.1 \%)$ received the second questionnaire by post. Of the 12 dropouts in the intervention group, four participants $(33.33 \%)$ had only attended the first session. Six participants $(50.00 \%)$ had attended the first two sessions and two parents-to-be (16.67\%) had dropped out after attending the third session. The dropout rates differed between groups, even tough not significantly $\left(\chi^{2}=1.89, \mathrm{p}=0.16\right)$.

Of the participants in the intervention group, $15 \%$ dropped out. In the control group, $23.7 \%$ of the participants dropped out.

The participants in the control group were significantly more often married than the participants in the intervention group $(\mathrm{T}=2.25, \mathrm{p}=0.02)$. The participants in the control group had significantly higher education levels $(\mathrm{T}=2.09, \mathrm{p}$ $=0.03)$ and sought help from a midwife $(\mathrm{T}=2.59, \mathrm{p}=$ $0.011)$ significantly more often.

\section{Differences in quality of relationship}

The intervention group had significantly more prenatal conflicts than the control group $(T=2.24, p=0.02$, Table 2 ). In contrast, the control group showed significantly higher levels of prenatal social support compared to the intervention group $(Z=-2.40, p=0.01$, Table 2$)$. The level of education was weakly associated with the social support score $(\mathrm{r}=0.18, \mathrm{p}=0.43)$. Education levels affected mean QRI prenatal social support scores $(\mathrm{F}(5,120)=3.17, \mathrm{p}=$ 0.01). An ANOVA was performed in order to explore the impact of education level on quality of relationship. Different intergroup education levels explained $11 \%$ of the variance in the QRI prenatal social support score. This is the effect size, as indicated by the partial eta-squared $(\eta 2=$ $0.11)$. The groups did not significantly differ in depth of relationship $(\mathrm{T}=0.39, \mathrm{p}=0.69$, Table 2). Mean and median values for intergroup comparisons of relationship quality at baseline are shown in Table 2 .

After the intervention, the groups did not differ in the level of conflict $(Z=-0.90, p=0.36)$, the level of social support $(Z=-1.26, p=0.20)$, or the level of depth $(Z=$ $-1.22, p=0.22)$. In contrast to the control group $(Z=$ $-1.00, p=0.31)$, the intervention group showed a significant decrease in conflicts in a pre-post comparison $(\mathrm{Z}=$ $-3.28, p=0.00$; Fig. 2). Regarding this decrease, a mixed ANOVA result showed that there was a significant decrease over time $(F(1,123)=8.31, \mathrm{p}=0.005$, eta-squared $=0.06)$ and there was a trend that intervention group reported higher conflicts than control group $(F(1,123)=3.225$, $\mathrm{p}=$ 0.075 , eta-squared $=0.03)$, but the group $\mathrm{x}$ time effect was not significant $(F(1,123)=2.175, \mathrm{p}=0.143$, eta-squared $=$ $0.02)$, which means the decrease in conflicts over time was 
Table 1. Sample descriptive statistics-by intervention status.

\begin{tabular}{|c|c|c|c|c|c|c|}
\hline & \multicolumn{2}{|c|}{$\begin{array}{l}\text { Total Sample } \\
n=126 \\
(63 \text { couples })\end{array}$} & \multicolumn{2}{|c|}{$\begin{array}{l}\text { Intervention } \\
\text { Group }\end{array}$} & \multicolumn{2}{|c|}{$\begin{array}{l}\text { Control } \\
\text { Group }\end{array}$} \\
\hline & $n$ & $\%$ & $n$ & $\%$ & $n$ & $\%$ \\
\hline \multicolumn{7}{|l|}{ Sex } \\
\hline Female & 63 & 50 & 34 & 50.0 & 29 & 50.0 \\
\hline Male & 63 & 50 & 34 & 50.0 & 29 & 50.0 \\
\hline \multicolumn{7}{|l|}{ Employment* } \\
\hline Employed & 118 & 93.7 & 66 & 97.1 & 52 & 89.7 \\
\hline Unemployed & 7 & 5.6 & 2 & 2.9 & 5 & 8.6 \\
\hline \multicolumn{7}{|l|}{ Annual gross income per person* } \\
\hline$<€ 15,000$ & 19 & 15.1 & 7 & 10.3 & 12 & 20.7 \\
\hline$€ 15-30,000$ & 25 & 19.8 & 14 & 20.6 & 11 & 19.0 \\
\hline$€ 30-60,000$ & 60 & 47.6 & 34 & 50.0 & 26 & 44.8 \\
\hline$€ 60-90,000$ & 10 & 7.9 & 7 & 10.3 & 3 & 5.2 \\
\hline over $€ 90,000$ & 10 & 7.9 & 4 & 5.9 & 6 & 10.3 \\
\hline \multicolumn{7}{|l|}{ Highest completed education } \\
\hline Lower secondary education & 1 & 0.8 & 0 & 0.0 & 1 & 1.7 \\
\hline Vocational school & 5 & 4.0 & 3 & 4.4 & 2 & 3.4 \\
\hline Upper secondary education & 21 & 16.7 & 16 & 23.5 & 5 & 8.6 \\
\hline Short-cycle tertiary education & 3 & 2.4 & 3 & 4.4 & 0 & 0.0 \\
\hline University of applied sciences & 18 & 14.3 & 10 & 14.7 & 8 & 13.8 \\
\hline University & 78 & 61.9 & 36 & 52.9 & 42 & 72.4 \\
\hline \multicolumn{7}{|l|}{ Relationship status } \\
\hline Partnership (joint place of residence) & 63 & 50.0 & 42 & 61.8 & 21 & 36.2 \\
\hline Partnership (no joint place of residence) & 7 & 5.6 & 0 & 0.0 & 7 & 12.1 \\
\hline Married (joint place of residence) & 56 & 44.4 & 26 & 38.2 & 30 & 51.7 \\
\hline \multicolumn{7}{|l|}{ Attachment patterns* } \\
\hline Secure & 88 & 69.8 & 47 & 69.1 & 41 & 70.7 \\
\hline Fearful-avoidant & 19 & 15.11 & 9 & 13.2 & 10 & 17.2 \\
\hline Preoccupied & 14 & 11.1 & 9 & 13.2 & 5 & 8.6 \\
\hline Dismissing & 4 & 3.2 & 2 & 2.9 & 2 & 3.4 \\
\hline \multicolumn{7}{|l|}{ Help by a midwife } \\
\hline Sought help & 106 & 84.1 & 52 & 76.5 & 54 & 93.1 \\
\hline Did not seek help & 20 & 15.9 & 16 & 23.5 & 4 & 6.9 \\
\hline
\end{tabular}

$n$, number of participants; $\%$, percent of total participants.

*Cases do not add up to 126 due to missing values.

not significantly different by intervention satus. No differences were found in either the perceived depth or support dimension of the QRI.

Fig. 2 shows the QRI conflict score (higher values mean more conflicts) separated for each group at baseline (prenatal) and after childbirth (postnatal).

\section{Postnatal differences in coparenting}

There were no group differences on the overall coparenting score $(Z=-0.115, p=0.908)$. Similar results were found for mothers $(Z=-0.028 p=0.978)$ and fathers $(Z=$ $-0.235, \mathrm{p}=0.814)$ independently.

Fathers achieved higher scores on the CPRS subscale "endorsing partner parenting" than mothers in both the intervention group $(Z=-2.93, p=0.003)$ and the control group $(Z=-2.25, p=0.024)$. No other gender differences in the CPRS subscales were found. Comparing the overall coparenting score between fathers and mothers revealed a significant difference between coparenting functioning $(\mathrm{T}=$ $2.30, \mathrm{p}=0.023)$. Fathers showed a mean of $4.42(\mathrm{SD}=$ $0.40)$ and mothers showed a mean of $4.23(\mathrm{SD}=0.54)$ in coparenting functioning.

\section{Differences in pre- and postpartum depression scores}

Although there were slight differences between pretest and post-test, the intervention group as well as the control group showed no significant change in their prenatal and postnatal EPDS scores (intervention group: $\mathrm{Z}=-0.83$, 
Table 2. Mean and median values for intergroup comparisons of relationship quality at baseline.

\begin{tabular}{lccc}
\hline & $\begin{array}{l}\text { Intervention Group } n=68 \\
(34 \text { couples })\end{array}$ & $\begin{array}{l}\text { Control Group } n=58 \\
(29 \text { couples })\end{array}$ & $\mathrm{p}$ \\
\hline $\begin{array}{l}\text { Conflicts prenatal } \\
\text { Mean (SD) }\end{array}$ & $2.01(0.41)$ & $1.85(0.41)$ & $0.02^{\mathrm{a}}$ \\
$\quad$ Median & 2.00 & 1.92 & \\
Depth prenatal & $3.39(0.34)$ & $3.37(0.32)$ & $0.69^{\mathrm{a}}$ \\
$\quad$ Mean (SD) & 3.50 & 3.33 & \\
$\quad$ Median & $3.39(0.36)$ & $3.52(0.39)$ & $0.01^{\mathrm{b}}$ \\
$\quad \begin{array}{l}\text { Social Support prenatal } \\
\text { Mean (SD) }\end{array}$ & 3.43 & 3.57 & \\
Median & &
\end{tabular}

a T-test; ${ }^{b}$ Mann-Whitney $U$ Test.

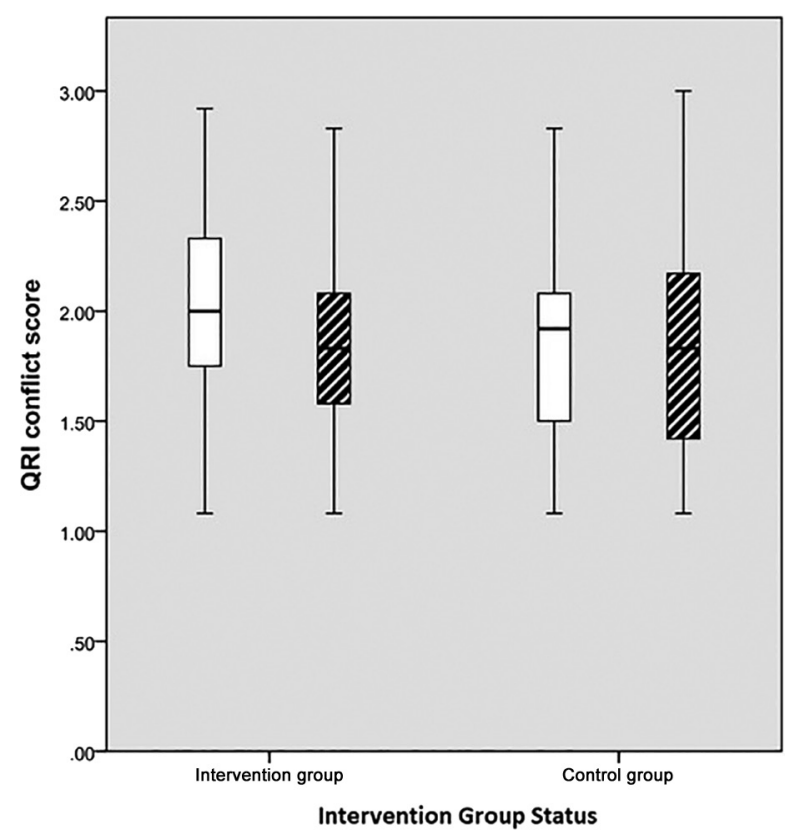

Fig. 2. Differences in prenatal and postnatal QRI (Quality of Relationship Inventory) conflict scores by intervention status.

The first two box plots show the differences in pre- and postnatal QRI conflict scores in the intervention group, and the last two box plots show the differences in preand postnatal QRI conflict scores in the control group. The white box plot in each group indicates prenatal QRI conflict scores and a black stiped box plot indicates postnatal QRI conflict scores. The mean and range of postnatal QRI conflict scores decreased in the intervention group compared to prenatal QRI conflict scores. The intervention group showed a significant decrease in conflicts in the pre-post comparison. On the contrary, in the control group, the variation in QRI conflict scores became greater after childbirth, which indicates some parents faced higher risks of interparental conflict in the postnatal period compared to the prenatal period.

$p=0.40$; control group: $Z=-0.86, p=0.38$ ). The mean of the EPDS prenatal score in the intervention group was 5.61 $(\mathrm{SD}=4.10$; median $=5.00)$ and $6.13(\mathrm{SD}=4.22$; median $=$
5.00) in the control group. The mean of the EPDS postnatal score in the intervention group was $5.97(\mathrm{SD}=4.51$; median $=5.00)$ and $5.53(\mathrm{SD}=3.71$; median $=4.50)$ in the control group. Neither fathers $(Z=-0.43, p=0.68)$ nor mothers $(Z=-0.31, p=0.75)$ showed a significant increase between the prenatal and postnatal EDPS scores. The mean of the EPDS prenatal score of fathers was $4.30(\mathrm{SD}=2.87$; median $=4.00)$ and $7.41(\mathrm{SD}=4.64$; median $=6.00)$ of mothers. The mean of the EPDS postnatal score of fathers was $4.34(\mathrm{SD}=3.00$, median $=4.00)$ and $7.19(\mathrm{SD}=4.65$; median $=7.00$ ) of mothers.

Rather, there was a significant negative correlation between the coparenting functioning CPRS scores and the postnatal EPDS scores among both fathers $(\mathrm{r}=-0.34, \mathrm{p}=$ $0.00)$ and mothers $(r=-0.30, p=0.01)$. Additionally, a negative correlation between the overall coparenting score and the postnatal EPDS score was found in the total sample $(\mathrm{r}=-0.37, \mathrm{p}=0.00)$.

\section{Postnatal differences in mutual support}

Postnatal individual delegated dyadic coping was positively correlated with group $(\mathrm{r}=0.18, \mathrm{p}=0.01)$, prenatal own delegated dyadic coping $(r=0.44, p=0.00)$, education level $(r=0.16, p=0.03)$, depth of relationship during pregnancy $(r=0.21, p=0.00)$, and the hiring a midwife around birth $(r=0.12, p=0.08)$.

The ordinary least-squares regression result showed that postnatal own delegated dyadic coping was predicted by group $(\beta=0.25, \mathrm{p}=0.00)$ and remained significant after controlling for education level $(\beta=0.18, \mathrm{p}=0.01)$, prenatal couple relationship depth $(\beta=0.20, \mathrm{p}=0.00)$, and the hiring of a midwife $(\beta=0.18, \mathrm{p}=0.02)$. Parents' postnatal own delegated dyadic coping was also predicted by prenatal own delegated dyadic coping $(\beta=0.40, \mathrm{p}=0.00)$. These results are summarized in Table 3 .

\section{Discussion}

Our coparenting intervention yielded several noteworthy findings. The aim of this intervention for expectant parents was to ease the transition to parenthood by estab- 
Table 3. Multivariate regression analysis for parents' own delegated dyadic coping after childbirth (mutual support).

\begin{tabular}{|c|c|c|c|c|}
\hline \multirow{2}{*}{$\begin{array}{l}\text { Parents' own delegated dyadic } \\
\text { coping (wave 2) }\end{array}$} & \multirow{2}{*}{ Beta } & \multirow{2}{*}{$\mathrm{p}$} & \multicolumn{2}{|c|}{$95 \%$ CI for B } \\
\hline & & & Lower bound & Upper bound \\
\hline Intervention status & 0.25 & 0.00 & 0.32 & 1.35 \\
\hline $\begin{array}{l}\text { Parents' own delegated dyadic } \\
\text { coping (wave 1) }\end{array}$ & 0.40 & 0.00 & 0.28 & 0.61 \\
\hline Education & 0.18 & 0.01 & 0.03 & 0.32 \\
\hline $\begin{array}{l}\text { Parents' depth of relationship } \\
\text { (wave 1) }\end{array}$ & 0.20 & 0.00 & 0.26 & 1.79 \\
\hline Midwifery care & 0.18 & 0.02 & 0.13 & 1.55 \\
\hline
\end{tabular}

$\mathrm{R}^{2}: 0.32$; adjusted $\mathrm{R}^{2}: 0.30$.

lishing an effective and strong coparenting alliance that could prevent both postpartum conflict and depression. The intervention was effective in strengthening relationship quality and mutual support of parents that have been in the intervention group. The intervention showed no effect on coparenting functioning, beside the gender difference. Neither of the two groups showed a significant increase in the depression score over time.

Despite the well-known exhaustion that parents experience after birth, parents in the intervention group managed to reduce their conflicts over time, possibly by learning to avoid attributing their distress to the relationship (Killien 1998; Bryan 2002). de Montigny and Lacharité (2004) reported an increase in interparental conflict in interventions that focus only on one parent, which suggests it is important to focus on both parents in intervention studies. Although we identified a decrease in conflicts, a very recent systematic review about the impact of coparenting interventions on paternal coparenting behaviour by Pilkington et al. (2019) surprisingly showed that interparental conflict was not significantly reduced in the reviewed studies.

Moreover, parents in the intervention group had higher scores in providing helping hands to relieve their respective partners' stress than parents in the control group. This significant association between group and parents' postnatal delegated coping skills still existed after controlling for education level, prenatal couple relationship depth, and the hiring of a midwife. These results underscore the need for interventions that strengthen the conflict management skills as well as coping skills of couples who are transitioning to parenthood. In the long run, conflict management as a determinant of relationship quality affects child development. Disagreements between couples and a lack of coping skills negatively impact child development, often leading to behaviour problems (Block et al. 1981). Previous research has reported a link between interparental conflict and child behaviour problems (Belsky 1984; Gable et al. 1994; McHale and Rasmussen 1998). Interventions supporting the transition to parenthood work protectively by creating a healthy environment for the child (Kapusta et al. 2017).

We found no increase in depression scores between the prenatal and postnatal measurements. Feinberg (2002) highlighted that among couple support approaches, coparenting support has the most powerful impact on parental adjustment and postnatal depression. The control group showed a high level of antenatal support that may have buffered postpartum depression in our study. It remains unknown whether the lack of a significant increase in depression scores in the intervention group is due to our early intervention programme. A follow-up study is needed to investigate if a significant difference in depression occurred up to one year postpartum.

Furthermore, the intervention group showed significantly lower levels of prenatal support than the control group. It can be assumed that highly functioning couples felt less need for an intense intervention and preferred to join the booklet-based control group. Expectant parents who are generally lower-functioning in support, conflict management, and stress management may have had an increased desire to join the intervention group. Additionally, there were no significant differences in the CPRS subscales between groups, which might be due to the groups' different starting points in prenatal support scores.

Feinberg and Kan (2008) found that their psychosocial coparenting prevention programme for expectant parents had a greater positive impact on less-educated parents and on families with insecurely attached fathers than it did on others. Our study's sample was disproportionately highly educated, with $62 \%$ of the participants holding a university degree. However, the distribution of attachment patterns in our sample was consistent with the general distribution of adult attachment styles (Mickelson et al. 1997), thus indicating a healthy total sample in terms to attachment. Whether our programme effects would be stronger in a lesseducated and more insecurely attached sample of participants merits investigation.

The dropout rate of $23.9 \%$ in the control group may be due to the weak attachment between participants and the study team, reflecting a low sense of belonging. Other explanations include unmet expectations about content of the study or a lack of interest in repeatedly filling out questionnaires. In order to minimize the loss of participants, we 
obtained information that would allow future contact by the study team (Song and Chung 2010).

The early dropout rates in the intervention group may be due to conflicting expectations about the coparenting intervention. However, no one dropped out after the fourth session, suggesting that parents subjectively benefitted from the course. Nevertheless, the actual causes of the dropouts remain unclear.

The significance of father involvement in childcare is stressed throughout the literature. Higher cognitive functioning, less emotional stress, fewer behaviour problems, more positive peer relations, and higher social competence are found in infants with highly interacting fathers (McBride et al. 2005; King and Sobolewski 2006; Formoso et al. 2007). Thus, the inclusion of both women and men in interventions is well justified. Additional research has shown that fathers' support in childrearing positively influences mothers' health as well as parenting stress level and consequently children's well-being (Gjerdingen et al. 1991; Nomaguchi et al. 2017). One of our additional outcomes showed a negative correlation between coparenting and postpartum depression scores. Feinberg et al. (2016) demonstrated that supportive coparenting has preventive effects for maternal mental health. Increased maternal depressive symptoms are linked with steeper declines in coparenting support and father involvement over time (Mallette et al. 2020), meaning that shared childcare and mutual support may buffer against mental health problems.

The quality of marital relationships and parenting alliances is significantly associated with father involvement in childcare (Feldman et al. 1983). Hence, strengthening the relationship between mothers and fathers can improve fathers' involvement (Formoso et al. 2007). These results demonstrate the importance of a supportive coparental relationship, which may be cultivated through a coparenting intervention. Our male participants scored higher on the coparenting subscale, "Endorse Partner Parenting," and the overall coparenting score. These findings suggest that our programme may have fostered father involvement in both the control and intervention groups. Nevertheless, future studies should consider adapting coparenting sessions to meet mothers' needs in order to reduce the difference in coparenting functioning.

The prenatal couple education programme focusing on Japanese couples done by Takeishi et al. (2019) and our coparenting intervention were both based on the Family Foundations programme of Feinberg (2002). In both cases the programme was adapted to meet the needs of the respective culture. Takeishi et al. (2019) described Japanese people as being shy or embarrassed about sharing their opinions. This is different to Austrian couples sharing their experiences in groups as there were lively discussions in our intervention groups of up to 6 couples. Therefore, each Japanese class included only one or two couples. The class size for European and American couples was much bigger, thereby creating lively discussions. However,
Japanese couples who were in the intervention group showed a significant positive effect on coparenting support scores at one month after childbirth. Moreover, the intervention had a positive effect on child adjustment (Takeishi et al. 2019). It is unclear why Japenese couples showed better results in coparenting support, if this is due to a different focus during the intervention or if it can be related to the differences in culture, the earlier timepoint of evaluation, or the approach to only instruct 1-2 couples in each session. Future studies are needed to shed more light on these aspects.

There are limitations and strengths in the present stsudy. The first weakness concerns the study design. Due to the design of a controlled case-control study, the study was prone to some form of selection bias (Thiese 2014). In future studies this kind of bias could be prevented by using a randomized, case-controlled study design. However, according to Thiese (2014), the choice of study design is only one component of successful research. Proper execution and detailed reporting are criteria of high-quality research that are important if the study is to provide sound conclusions (Thiese 2014). The second weakness refers to the sample size. A larger sample would be more representative and possibly yield more significant differences. Moreover, the information included in our booklet for the intervention group may have diminished the intervention effect. Therefore, future studies should reduce the amount of information in such booklets. Additionaly, the participants in the intervention group had to fill out the second questionnaire immediately after the last session. Control group participants who did not participate in the postpartum meeting had a longer time frame to fill out the questionnaire. This latter group received the questionnaire by post and had to send it back within two weeks, thereby enabling the participants to fill out the questionnaire when they were in a good mood and under less time pressure. If some participants in the intervention group had been having a bad day during the last session, their bad mood may have had a negative influence on their response behaviour. Another limitation of the sample was the participants' high education levels, possibly leading to more non-significant results.

In contrast to these limitations, some strengths of this study merit emphasis. This educational intervention on how partners can support each other around the time of birth and properly solve conflicts is able to enhance prenatal care in the German-speaking world. Moreover, individualized data were collected over time, a strength of primary research (Thiese 2014). Some expectant parents mentioned that they really enjoyed the idea of a couple-focused intervention as preparation for parenthood. Men often feel excluded from prebirth preparation classes, criticise the lack of information about fatherhood, and easily feel unprepared, because most classes focus only on the mothers' interactions with their babies (Deave et al. 2008). The fact that our intervention addressed both parents-to-be is a strength. 
In conclusion, the results of this pilot study are promising. Our intervention seems to support a smooth transition to parenthood by reducing conflicts, strengthening dyadic coping skills and likely increasing father involvement. However, the effects were smaller than those observed in similar U.S. trials (Fagan 2008; Feinberg and Kan 2008; Doss et al. 2014; Jones et al. 2018). This may be due to a reduced number of intervention sessions, cultural differences, our smaller sample size, or other discussed methodological weaknesses.

Our evaluated intervention could serve as foundation for future programmes in the field of early intervention, especially in the German-speaking world. In this way, we would move one step closer towards improving coparenting behaviour and ultimately making a positive impact on the development of children. Future studies with a randomized design are needed to investigate the impact of such early interventions on the development of children.

\section{Conflict of Interest}

The authors declare no conflict of interest.

\section{References}

American Psychiatric Association (2013) Diagnostic and statistical manual of mental disorders, 5th ed., American Psychiatric Publishing, Arlington, Washington DC, USA.

Bartholomew, K. \& Horowitz, L.M. (1991) Attachment styles among young adults: a test of a four-category model. J. Pers. Soc. Psychol., 61, 226-244.

Belsky, J. (1984) The determinants of parenting: a process model. Child Dev., 55, 83-96.

Black, K. \& Lobo, M. (2008) A conceptual review of family resilience factors. J. Fam. Nurs., 14, 33-55.

Block, J.H., Block, J. \& Morrison, A. (1981) Parental agreementdisagreement on child- rearing orientations and gender-related personality correlates in children. Child Dev., 52, 965-974.

Bodenmann, G. (2008) Dyadisches Coping Inventar, Huber, Switzerland.

Brotherson, S.E. (2007) From partners to parents: couples and the transition to parenthood. IJCE, 22, 7-12.

Bryan, A.A. (2002) Couple relationships over the transition to parenthood: methodological issues in testing for an intervention effect. J. Fam. Nurs., 8, 201-220.

Condon, J.T., Boyce, P. \& Corkindale, C.J. (2004) The first-time fathers study: a prospective study of the mental health and wellbeing of men during the transition to parenthood. Aust. NZ. J. Psychiatry, 38, 56-64.

Cowan, C.P., Cowan, P.A., Heming, G., Garrett, E., Coysh, W.S., Curtis-boles, H. \& Boles, A.J. 3rd (1985) Transitions to parenthood: his, hers, and theirs. J. Fam. Issues, 6, 451-481.

Cowan, C.P. \& Cowan P.A. (1988) Who does what when partners become parents: implications for men, women, and marriage. Marriage Fam. Rev., 12, 105-131.

Cowan, C.P. \& Cowan P.A. (1995) Interventions to ease the transition to parenthood: why they are needed and what they can do. J. Fam. Relat., 44, 412-423.

Cox, J.L., Holden, J.M. \& Sagovsky, R. (1987) Detection of postnatal depression. Development of the 10-item Edinburgh Postnatal Depression Scale. Br. J. Psychiatry, 150, 782-786.

de Montigny, F. \& Lacharite, C. (2004) Fathers'perceptions of the immediate postpartal period. J. Obstet. Gynecol. Neonatal Nurs., 33, 328-339.

Deave, T., Johnson, D. \& Ingram, J. (2008) Transition to parent- hood: the needs of parents in pregnancy and early parenthood. BMC Pregnancy Childbirth, 8,30 .

Diem-Wille, G. (2018) The baby as catalyst for unconscious conflicts of the parents. Contribution to a psychoanalytically oriented parent-infant therapy. Infant Obs., 2, 17-32.

Doss, B.D., Cicila, L.N., Hsueh, A.C., Morrison, K.R. \& Carhart, K. (2014) A randomized controlled trial of brief coparenting and relationship interventions during the transition to parenthood. J. Fam. Psychol., 28, 483-494.

Elliott, S.A., Leverton, T.J., Sanjack, M., Turner, H., Cowmeadow, P., Hopkins, J. \& Bushnell, D. (2000) Promoting mental health after childbirth: a controlled trial of primary prevention of postnatal depression. Br. J. Clin. Psychol., 39, 223-241.

Fagan, J. (2008) Randomized study of a prebirth coparenting intervention with adolescent and young fathers. Fam. Relat., 57, 309-323.

Falconier, M.K. \& Kuhn, R. (2019) Dyadic coping in couples: a conceptual integration and a review of the empirical literature. Front. Psychol., 10, 571.

Falconier, M.K., Nussbeck, F. \& Bodenmann, G. (2013) Dyadic coping in Latino couples: validity of the Spanish version of the Dyadic Coping Inventory. Anxiety Stress Coping, 26, 447-466.

Feinberg, M.E. (2002) Coparenting and the transition to parenthood: a framework for prevention. Clin. Child Fam. Psychol. Rev., 5, 173-195.

Feinberg, M.E., Brown, L.D. \& Kan, M.L. (2012) A multi- domain self-report measure of coparenting. Parent. Sci. Pract., 12, $1-21$.

Feinberg, M.E., Jones, D.E., Roettger, M.E., Hostetler, M.L., Sakuma, K.L., Paul, I.M. \& Ehrenthal, D.B. (2016) Preventive effects on birth outcomes: buffering impact of maternal stress, depression, and anxiety. Matern. Child Health J., 20, 56-65.

Feinberg, M.E. \& Kan, M.L. (2008) Establishing family foundations: intervention effects on coparenting, parent/infant wellbeing, and parent-child relations. J. Fam. Psychol., 22, 253-263.

Feinberg, M.E., Kan, M.L. \& Goslin, M.C. (2009) Enhancing coparenting, parenting, and child self-regulation: effects of family foundations 1 year after birth. Prev. Sci., 10, 276-285.

Feldman, S.S., Nash, S.C. \& Aschenbrenner, B.G. (1983) Antecedents of fathering. Child Dev., 54, 1628-1636.

Formoso, D., Gonzales, N.A., Barrera, M. Jr. \& Dumka, L.E. (2007) Interparental relations, maternal employment, and fathering in Mexican American families. J. Marriage Fam., 69, 26-39.

Gable, S., Crinc, K. \& Belsky, J. (1994) Coparenting within the family system: Influences on children's development. Fam. Relat., 43, 380-386.

Gjerdingen, D.K., Froberg, D.G. \& Fontaine, P. (1991) The effects of social support on women's health during pregnancy, labor and delivery, and the postpartum period. Fam. Med., 23, 370-375.

Hanington, L., Heron, J., Stein, A. \& Ramchandani, P. (2012) Parental depression and child outcomes: is marital conflict the missing link? Child Care Health Dev., 38, 520-529.

Harms, T. (2008) Emotionelle Erste Hilfe: BindungsförderungKrisenintervention-Eltern-Baby-Therapie, Leutner, Berlin, Germany.

Johnson, S.L. \& Jacob, T. (1997) Marital interactions of depressed men and women. J. Consult. Clin. Psychol., 65, 15-23.

Jones, D., Feinberg, M., Hostetler, M., Roettger, M., Paul, I.M. \& Ehrenthal, D.B. (2018) Family and child outcomes 2 years after a transition to parenthood intervention. Fam. Relat., 67, 270-286.

Kapusta, N.D. Philipp, A.C. \& Feinberg, M.E. (2017) Co-parenting during transition to parenthood: creating a healthy nurturing environment for children. Austin Child 
Adolesc. Psychiatry, 2, 1008.

Killien, M.G. (1998) Postpartum return to work: mothering stress, anxiety, and gratification. Can. J. Nurs. Res., 30, 53-66.

King, V. \& Sobolewski, J.M. (2006) Nonresident fathers' contributions to adolescent well-being. J. Marriage Fam., 68, 537-557.

König-Bachmann, M., Zenzmaier, C. \& Schildberger, B. (2019) Health professionals' views on maternity care for women with physical disabilities: a qualitative study. BMC Health Serv. Res., 19, 551.

Lee, J.Y., Knauer, H.A., Lee, S.J., MacEachern, M.P. \& Garfield, C.F. (2018) Father-inclusive perinatal parent education programs: a systematic review. Pediatrics, 142, e20180437.

Mallette, J.K., Futris, T.G., Oshri, A. \& Brown, G.L. (2020) Paternal support and involvement in unmarried fragile families: impacts on long-term maternal mental health. Fam. Process, 59, 789-806.

McBride, B.A., Schoppe-Sullivan, S.J. \& Ho, M. H. (2005) The mediating role of fathers' school involvement on student achievement. J. Appl. Dev. Psychol., 26, 201-216.

McHale, J.P. \& Rasmussen, J.L. (1998) Coparental and family group-level dynamics during infancy: early family precursors of child and family functioning during preschool. Dev. Psychopathol., 10, 39-59.

Mickelson, K.D., Kessler, R.C. \& Shaver, P.R. (1997) Adult attachment in a nationally representative sample. J. Pers. Soc. Psychol., 73, 1092-1106.

Miller, R.L., Pallant, J.F. \& Negri, L.M. (2006) Anxiety and stress in the postpartum: is there more to postnatal distress than depression? BMC Psychiatry, 6, 12.

Molgora, S., Fenaroli, V., Acquati, C., De Donno, A., Baldini, M.P. \& Saita, E. (2019) Examining the role of dyadic coping on the marital adjustment of couples undergoing assisted reproductive technology (ART). Front. Psychol., 10, 415.

Moss, P., Bolland, G., Foxman, R. \& Owen, C. (1986) Marital relations during the transition to parenthood. J. Reprod. Infant Psyc., 4, 57-67

Nomaguchi, K., Brown, S.L. \& Leyman, T.M. (2017) Fathers' participation in parenting and maternal parenting stress: variation by relationship status. J. Fam. Issues, 38, 1132-1156.

O'Hara, M.W. \& Swain, A.M. (1996) Rates and risks of postpartum depression: a meta- analysis. Int. Rev. Psychiatr., 8, 37-54.

O'Hara, M.W. \& McCabe, J.E. (2013) Postpartum depression: current status and future directions. Annu. Rev. Clin. Psychol., 9, 379-407.

Parfitt, Y.M. \& Ayers, S. (2009) The effect of post-natal symptoms of post-traumatic stress and depression on the couple's relationship and parent: baby bond. J. Reprod. Infant Psyc., 27, 127-142.

Paulson, J.F. \& Bazemore, S.D. (2010) Prenatal and postpartum depression in fathers and its association with maternal depres- sion: a meta-analysis. JAMA, 303, 1961-1969.

Pilkington, P., Rominov, H., Brown, H.K. \& Dennis, C.L. (2019) Systematic review of the impact of coparenting interventions on paternal coparenting behaviour. J. Adv. Nurs., 75, 17-29.

Ramchandani, P., Stein, A., Evans, J. \& O'Connor, T.G.; ALSPAC study team (2005) Paternal depression in the postnatal period and child development: a prospective population study. Lancet, 365, 2201-2205.

Randall, A.K., Hilpert, P., Jimenez-Arista, L.E., Walsh, K.J. \& Bodenmann, G. (2015) Dyadic coping in the U.S.: psychometric properties and validity for use of the English version of the dyadic coping inventory. Curr. Psychol., 35, 570-582.

Reiner, I., Beutel, M., Skaletz, C., Brahler, E. \& Stobel-Richter, Y. (2012) Validating the German version of the quality of relationship inventory: confirming the three-factor structure and report of psychometric properties. PLoS One, 7, e37380.

Robakis, T.K., Williams, K.E., Crowe, S., Lin, K.W., Gannon, J. \& Rasgon, N.L. (2016) Maternal attachment insecurity is a potent predictor of depressive symptoms in the early postnatal period. J. Affect. Disord., 190, 623-631.

Shapiro, A.F. \& Gottman, J.M. (2005) Effects on marriage of a psycho-communicative-educational intervention with couples undergoing the transition to parenthood, evaluation at 1-year post intervention. J. Fam. Commun., 5, 1-24.

Song, J.W. \& Chung, K.C. (2010) Observational studies: cohort and case-control studies. Plast. Reconstr. Surg., 126, 22342242.

Takeishi, Y., Nakamura, Y., Kawajiri, M., Atogami, F. \& Yoshizawa, T. (2019) Developing a prenatal couple education program focusing on coparenting for Japanese couples: a quasi-experimental study. Tohoku J. Exp. Med., 249, 9-17.

Thiese, M.S. (2014) Observational and interventional study design types; an overview. Biochem. Med. (Zagreb), 24, 199-210.

Tomlinson, P.S. (1996) Marital relationship change in the transition to parenthood: a reexamination as interpreted through transition theory. J. Fam. Nurs., 2, 286-305.

Tynes, S.R. (1990) Educational heterogamy and marital satisfaction between spouses. Soc. Sci. Res., 19, 153-174.

Wiik, K.A., Bernhardt, E., Noack, T. \& Teachman, J. (2009) A study of commitment and relationship quality in sweden and norway. J. Marriage Fam., 71, 465-477.

Winkler, N., Kroh, M. \& Spiess, M. (2006) Entwicklung einer deutschen Kurzskala zur zweidimensionalen Messung von sozialer Erwünschtheit, Deutsches Institut für Wirtschaftsforschung, Berlin, Germany.

World Health Organization (2010) mhGAP Intervention guide for mental, neurological and substance use disorders in nonspecialized health settings.

https://www.who.int/mental_health/publications/mhGAP intervention guide/en/

[Accessed: August 18, 2020]. 